\title{
Acessibilidade na formação de professores a distância: um estudo de caso das dimensões técnica e pedagógica
}

\author{
Lucila Maria Costi Santarosa, NIEE/UFRGS - lucila.santarosa@ufrgs.br \\ Débora Conforto, NIEE/UFRGS - deboraconforto@gmail.com \\ Maristela Compagnoni Vieira, NIEE/UFRGS, CESUCA - maricompagnoni@gmail.com \\ Fernanda Schneider, NIEE/UFRGS, SMED PMPA - ferscsc@ yahoo.com.br \\ Jean Felipe Patikowski Cheiran - NIEE/UFRGS, UNIPAMPA - jfpcheiran@ gmail.com
}

Resumo: Três elementos podem ser mencionados com destaque no que tange à caraterização da educação contemporânea: a crescente inserção das tecnologias digitais nos ambientes pedagógicos, a ascensão da modalidade de ensino a distância e a inclusão de pessoas com deficiência em instituições não especiais. Enquanto esses elementos podem ser considerados isoladamente como novos desafios, a combinação de todos eles requer um conjunto de esforços que viabilizem a qualidade da experiência e a permanência de todos os sujeitos no sistema educacional. Nessa perceptiva, este estudo apresenta a percepção de sujeitos com e sem deficiência, envolvidos em um curso de formação de professores na modalidade a distância, quanto à contribuição de dois tipos de estratégias: técnicas, voltadas à promoção da acessibilidade, e pedagógicas, destinadas à adaptação curricular. Os resultados sugerem a validade de tais ações, mas apontam para fragilidades no ambiente virtual de aprendizagem utilizado.

Palavras-chaves: EAD, Formação de Professores, Tecnologias Digitais na Educação, Acessibilidade, Inclusão.

Accessibility in Teacher Education Distance Learning: a case study of the technical and pedagogical dimensions

Abstract: Three elements can be mentioned especially regarding the characterization of contemporary education: the increasing integration of digital technologies in educational environments, the rise of the distance education mode and the inclusion of people with diverse needs in regular institutions. While these elements can alone be considered as new challenges, the combination of them all requires a set of technical and methodological efforts that allow the quality of the experience and the permanence of all subjects in the educational training system. From this perceptive, this study presents the perception of people with and without diverse needs, who are involved in a distance teacher education course, in relation to the contribution of two kinds of strategies: technical and pedagogical. The technical strategy focuses on the promotion of accessibility, and pedagogical one addresses the adaptations of the course program. The results suggest that these actions are valid, but also indicate some weaknesses in the learning virtual environment which was used.

Key Words: Distance Education, Teacher Education, Digital Technologies in Education, Accessibility, Inclusion. 


\section{INTRODUÇÃO}

No cenário educacional brasileiro, o foco da discussão sobre a Educação Especial explicita um deslocamento, da escola ou do aluno com deficiência, para os currículos, métodos, técnicas, recursos e organização específicos com vistas ao atendimento das demandas dos estudantes com deficiência. Uma das demandas elencadas na Declaração de Salamanca (UNESCO, 1994) e, posteriormente, nas políticas públicas brasileiras de inclusão escolar, diz respeito à existência de um currículo especial para atender às diferentes necessidades da diversidade humana: a adoção de sistemas mais flexíveis e adaptativos, capazes de considerar amplamente as diferentes necessidades dos estudantes irá contribuir tanto para o sucesso educacional quanto para a inclusão.

No que diz respeito à formação de professores para a utilização de tecnologias na Educação, práticas pedagógicas inovadoras acontecem quando as instituições se propõem a repensar e a transformar sua estrutura cristalizada em uma configuração curricular flexível, dinâmica e articulada com a Sociedade da Informação/Conhecimento. Lançar mão desses conceitos em um curso de formação que busca instrumentalizar professores em serviço para a utilização de tecnologias acessíveis na escola inclusiva, sobretudo quando o próprio público da formação passa a ser constituído por pessoas com e sem deficiência, é fundamental para a construção de um currículo e de uma dinâmica pedagógica capazes de atender às necessidades de um conjunto de educadores, de acordo com as realidades vivenciadas nos diferentes estados e escolas brasileiras envolvidas na formação, principalmente, quando o foco se coloca sobre os desafios da Educação Inclusiva.

Valente (1999: 19) afirma que "os assuntos desenvolvidos durante um curso devem ser escolhidos pelos professores, de acordo com o currículo e a abordagem pedagógica adotados pela escola". Ainda neste sentido, de acordo com Rezende (2004), o atendimento a uma rede complexa, composta por estudantes com e sem deficiência, deve amparar-se em um conceito-guia baseado em três aspectos: a concepção de ensino e aprendizagem, neste caso, o sócio-interacionismo, proposto por Vygotsky (1994); as necessidades e interesses dos alunos (construir conhecimentos, desenvolver habilidades e competências de uma dada área) e a intenção pedagógica inerente ao curso (os objetivos de aprendizagem no contexto político pedagógico da instituição).

Com o propósito de reafirmar o potencial das tecnologias digitais de informação e de comunicação na implementação de políticas públicas em prol do atendimento especializado para a diversidade humana, o Curso Tecnologias Digitais Acessíveis observa o professor como peça central para a concretização da inclusão sociodigital de alunos com deficiência. A formação de 180 horas é estruturada em seis módulos, que operam em complementaridade para que os educadores conquistem a fluência digital: de meros usuários de tecnologia passando a exercer o papel de autores e protagonistas da inclusão. O curso é proposto e gerenciado pela equipe de pesquisa do Núcleo de Informática na Educação Especial (NIEE) da Universidade Federal do Rio Grande do Sul (UFRGS), um grupo de investigadores que, atento às diferentes modificações do cenário educacional brasileiro, tem produzido tempo e espaço de formação em permanente reestruturação para responder às demandas da contemporânea escola tecnológica e inclusiva.

Nos últimos 14 anos, o curso já atendeu cerca de 12.000 professores oriundos da rede pública brasileira e de países da América Latina, América Central e Espanha, contando com pessoas com variadas deficiências físicas e sensoriais, tanto entre os professores cursistas quanto entre tutores e formadores. Diante deste cenário, uma série de estratégias 
técnicas e pedagógicas foram implementadas, com vistas à promoção ao acesso e à qualidade da formação para todos os professores cursistas, o que é avaliado neste estudo, que apresenta as estratégias de acessibilidade técnica e pedagógica implementadas no curso, bem como a percepção dos sujeitos envolvidos quanto à sua validade e contribuição.

\subsection{Acessibilidade e Educação a Distância (EAD)}

A acessibilidade na modalidade a distância trata de assegurar princípios que garantam que qualquer usuário consiga utilizar de forma efetiva seus recursos, independentemente da sua condição de acesso e de suas especificidades físicas, cognitivas e sensoriais. Participantes de cursos na modalidade a distância podem, por exemplo, utilizar um computador que não esteja equipado com caixas de som; utilizar uma conexão com a Internet instável ou de velocidade reduzida ou, ainda, ter sua interação mediada por um leitor de tela. Essas e outras questões, entretanto, não podem constituir um impedimento para acesso à informação.

A usabilidade, por sua vez, aborda a qualidade de uso de uma interface de software, ou seja, o quão fácil ela é para seus usuários. Nielsen (1993) sugere cinco componentes básicos para usabilidade (facilidade de aprender, facilidade de lembrar, facilidade em evitar erros e lidar com erros, eficiência de uso, e satisfação no uso) que afetam todos os usuários, a despeito de suas tecnologias e limitações. Assim, observa-se que recomendações de acessibilidade e usabilidade devem ser aplicadas em conjunto para garantir a ampliação do acesso para todos os públicos e a qualidade na interação com conteúdos e funcionalidades.

Diversos estudos vêm sendo conduzidos nos últimos anos a fim de estabelecer recomendações e apresentar estratégias que garantam o acesso de estudantes e professores ou instrutores, com e sem deficiências, a cursos na modalidade a distância, com vistas a promover também uma maior qualidade e eficiência na interação com os ambientes virtuais e materiais didáticos. Bassani et al. (2010), Burgstahler (2001) e Bugstahler, Corrigan e McCarter (2004) desenvolvem estudos voltados à identificação das barreiras enfrentadas por sujeitos com deficiência em EAD e apresentam sugestões estratégicas pautadas em elementos como o Design de Interação e o Design Universal.

Frequentemente, investigações voltadas à acessibilidade em EAD direcionam-se especificamente para sujeitos cegos ou com baixa visão, como Estabel e Moro (2006) e Sonza e Santarosa (2003). Observa-se ainda que diferentes estudos identificam fragilidades na acessibilidade dos próprios AVAs nos quais os cursos são operacionalizados (Estabel e Moro, 2006; Santarosa et al. 2014), o que se reflete na demanda pelo desenvolvimento de adaptações nos ambientes existentes ou, ainda, no desenvolvimento de outros, totalmente projetados com a premissa da equidade de acesso (Santarosa et al., 2007), como nos casos dos AVAs Eduquito e Place, ambos desenvolvidos pelo NIEE/UFRGS.

\section{METODOLOGIA}

A pesquisa realizada caracterizou-se por ser descritiva, com abordagem qualiquantitativa, com base no levantamento de dados (GIL, 2002). Os resultados foram coletados por meio de um questionário digital composto por 17 questões, submetido a 
validações automáticas e manuais de acessibilidade, bem como por testes junto a sujeitos cegos e surdos antes da aplicação. Do total de 1064 professores cursistas para os quais o questionário foi enviado, foram obtidas 196 respostas. A amostra constituiu-se de 177 mulheres e 77 homens, com idade média de 41,6 anos. No que se refere à diversidade física e sensorial, $91 \%$ das pessoas manifestaram não apresentar nenhuma deficiência, enquanto 9\% declararam alguma deficiência como: baixa audição, deficiência física, baixa visão, surdez e cegueira.

O exame dos dados foi conduzido por meio da técnica de Análise de Conteúdo, conforme proposto por Bardin (2007) e Moraes (2003), o que permitiu verificar o surgimento de conceitos a partir da incidência de unidades de sentido presentes nos variados discursos examinados. Adicionalmente, as respostas dissertativas foram estudadas para identificação de incidentes críticos negativos durante o uso do sistema e sua consequente classificação como problemas de acessibilidade ou usabilidade, conforme recomendações de Nielsen (1993). Os dados são apresentados, principalmente, por meio de números percentuais, mais adequados para estudos com amostras superiores a 10 sujeitos, favorecendo a padronização dos dados (PREECE, ROGERS e SHARP, 2005).

\section{ADAPTAÇÕES DE ACESSO}

Os materiais do Curso em questão foram desenvolvidos com base em pesquisas brasileiras e internacionais sobre Acessibilidade à Web, em particular as Diretrizes de Acessibilidade para Conteúdo Web (WCAG) 2.0 (W3C, 2008), e nos estudos de heurísticas de usabilidade (Nielsen, 1993, 1994) e critérios ergonômicos de interfaces humanocomputador (BASTIEN, SCAPIN, 1993) para garantir o acesso de todos os professores cursistas ao conteúdo digital da formação. As estratégias de usabilidade e acessibilidade técnicas implementadas no curso estão sumarizadas no Quadro 1, e alguns destes elementos estão ilustrados na Figura 1.

Quadro 1: Sumarização das estratégias de usabilidade e acessibilidade

\begin{tabular}{|c|}
\hline Equivalente textual para todo conteúdo não textual. (Figura 1, B) \\
\hline Folhas de estilo para separação do conteúdo dos elementos de estilização. \\
\hline Uso exclusivo de tabelas para apresentação de dados tabulares, permitindo sua linearização. \\
\hline Conteúdos e funcionalidades integralmente acessíveis por teclado. \\
\hline Navegação linear, otimizada pela inserção de links- âncora para o conteúdo principal. (Figura 1, A) \\
\hline Retorno claramente identificado por links contextuais de "voltar" no final de cada página. (Figura 1, F) \\
\hline Repetição e ordenação de elementos significativos para propiciar navegação consistente. \\
\hline Escolha de cores pautada em recomendações rígidas de contraste, testadas por validadores automáticos. \\
\hline Esquemas de cores aplicados para auxiliar usuários surdos a reconhecer blocos de atividades. \\
\hline $\begin{array}{l}\text { Conteúdo externo ao material do curso incorporado às páginas, minimizando possíveis problemas de } \\
\text { acessibilidade e evitando que o aluno tenha sua atenção e sua concentração reduzidas. }\end{array}$ \\
\hline $\begin{array}{l}\text { Links internos de acordo com padrão Web (texto azul sublinhado) para facilitar o reconhecimento de } \\
\text { elementos clicáveis. (Figura } 1, \mathrm{~A} \text { ) }\end{array}$ \\
\hline
\end{tabular}

Destaca-se que todo o conteúdo textual do curso é disponibilizado, igualmente, por meio de vídeos em Língua Brasileira de Sinais - Libras (Figura 1 - D) e de áudios no formato mp3 (Figura 1 - C) cujo desenvolvimento não demanda estruturas sofisticadas: um computador minimamente equipado com fones de ouvido, microfone e softwares gratuitos para edição de áudio e vídeo permite o desenvolvimento de tais materiais. 


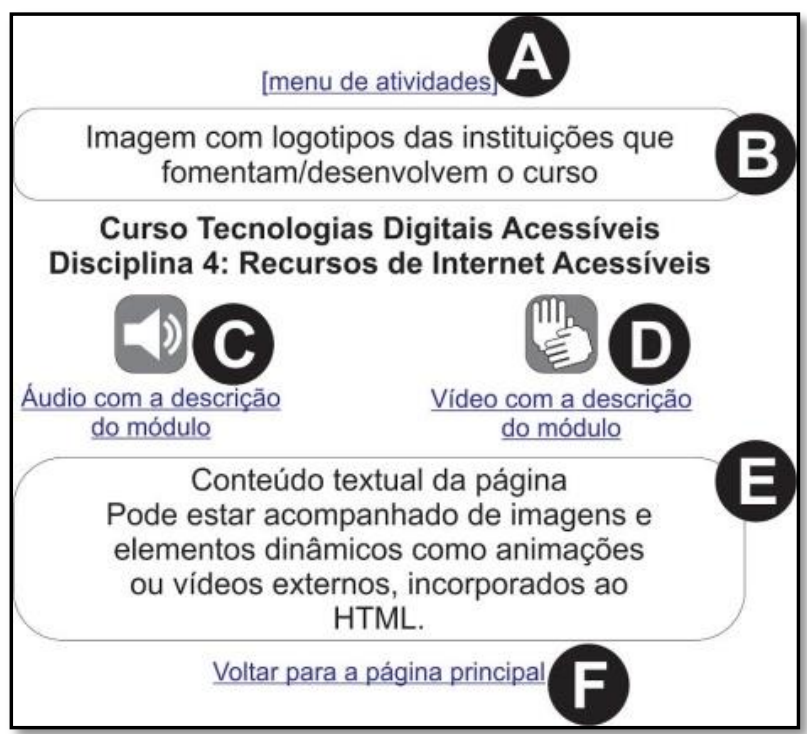

Figura 1: Recorte da interface de uma das páginas HTML que apresenta o conteúdo do curso

Os sujeitos participantes do Curso, a maioria dos quais sem deficiência alguma, afirmaram beneficiar-se dos recursos de acessibilidade disponibilizados no ambiente, como as descrições em áudio, por exemplo. Tais declarações referendam que a acessibilidade torna os sistemas melhores também para as pessoas que não têm deficiência (TATCHER, 2006). Os professores cursistas sem deficiência revelaram favorecerem-se dos recursos de acessibilidade em situações como: "às vezes, estou com a vista cansada; eu gosto do leitor de tela" e "o áudio com descrição das atividades foi um bom auxílio, quando eu necessitava fazer mais de uma atividade ao mesmo tempo". Todos os professores cursistas com alguma deficiência, que responderam à pesquisa, afirmaram que tais estratégias de acessibilidade contribuíram para sua permanência na formação.

As análises referentes à acessibilidade foram dividas em três planos: acesso ao ambiente e às ferramentas do Ambiente Virtual de Aprendizagem (AVA) Teleduc e acesso às páginas HTML desenvolvidas pela equipe de coordenação do curso. Sobre problemas de acesso específicos do ambiente virtual - Teleduc, 25\% dos entrevistados mencionaram ter dificuldades com uma ou mais ferramentas presentes na plataforma.

O cruzamento de dados permite asseverar que do grupo de pessoas que mencionou identificar fragilidades em alguma das ferramentas existentes no ambiente virtual, $29 \%$ também destacaram ter dificuldades no uso do computador e outros $12 \%$ apresentaram alguma deficiência. No primeiro caso, embora a plataforma Teleduc exiba um layout simples, identificamos que os problemas citados se relacionam a questões de usabilidade, ligadas à compreensão da interface por todos. Já no segundo, inferimos que barreiras de acessibilidade devem ser responsáveis por impedimentos às pessoas com deficiência.

Como forma de minimizar as barreiras de acessibilidade impostas para a interação síncrona do AVA, serviços disponíveis em outras plataformas são utilizados em complementariedade, como Skype e Hangouts, que suportam conversação por voz e por vídeo. 
No que diz respeito a dificuldades relacionadas às páginas HTML de descrição de atividades, produzidas pela equipe do curso, 91\% do total de sujeitos inqueridos referem que não enfrentam problemas de acesso, dos quais 17 são pessoas com deficiência.

A análise dos dados permitiu concluir que as avaliações mais negativas sobre a acessibilidade do curso estão relacionadas às interfaces oferecidas pelo AVA onde ocorrem as interações, e não às páginas HTML onde são disponibilizadas as atividades do curso, desenhadas sob as normas e diretrizes WCAG 2.0, conforme explicitado na Tabela 1.

Tabela 1: Proporção da dificuldade de acesso, conforme análise

Dificuldade de acesso ao Dificuldade de acesso às ambiente Teleduc páginas HTML $25 \%$ $9 \%$

\section{ADAPTAÇÕES CURRICULARES: A PROPOSTA DE FLEXIBILIZAÇÃO}

Com vistas a promover a flexibilização curricular, atendendo aos interesses e necessidades do público-alvo da formação, as disciplinas (ou módulos) foram divididas em blocos temáticos, nos quais são disponibilizadas diversas atividades. Para cada bloco, o professor cursista deve escolher, no mínimo, uma atividade para realizar. Uma vez que as tarefas apresentadas em cada bloco estão relacionadas a um conceito-chave, foi possível desenhar uma linha formativa, sem, no entanto, engessar as possibilidades de aprendizagem do professor. Na medida em que o cursista tem o poder de escolha quanto ao seu currículo de estudo, passa a ser estabelecida a corresponsabilidade de cada educador na construção de seu conhecimento no âmbito das tecnologias digitais acessíveis.

Para tornar mais clara para o leitor tal proposta de flexibilização curricular, apresentamos a dinâmica visualizando as estratégias em ação na disciplina de Introdução à Informática Acessível, conforme Figura 2.

Bloco A: Escolha e realize uma das 3 atividades

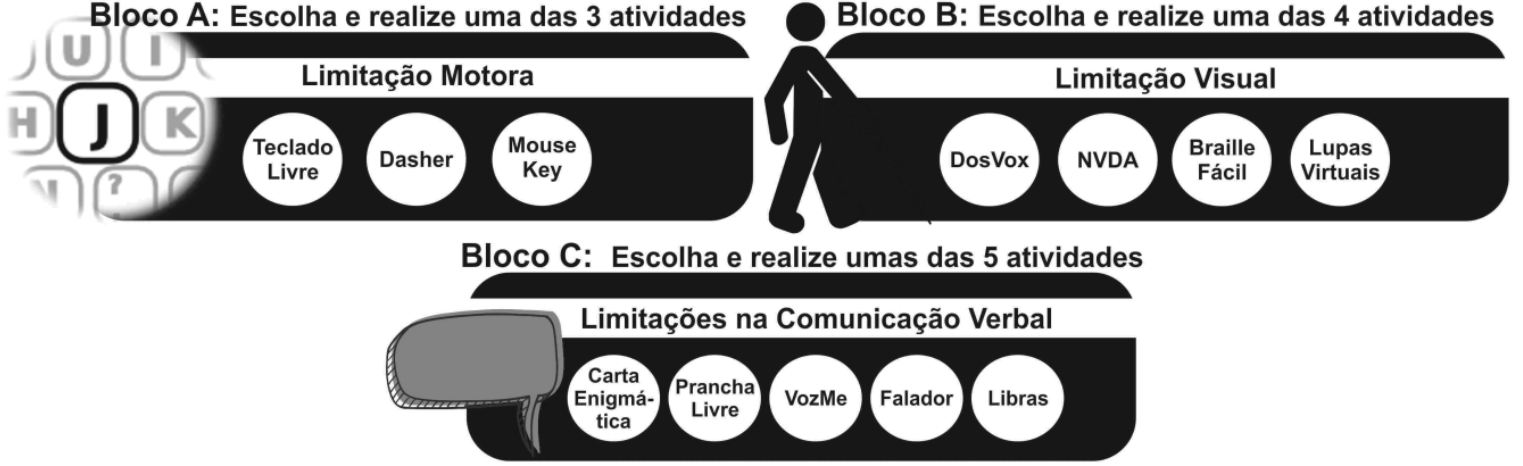

Figura 2: Proposta de flexibilização Curricular por meio de blocos temáticos de atividades

O campo de saber e a materialidade da Tecnologia Assistiva foram estruturados em três blocos temáticos, módulo em que ajudas técnicas foram apresentadas nas áreas de limitação motora, visual e na comunicação verbal. Os professores cursistas exploram os três blocos, realizando ao menos uma atividade para cada uma dos eixos propostos. O diferencial da possibilidade de escolha está no aprofundamento do eixo temático no qual o 
processo de inclusão se coloca de forma mais desafiadora para o professor cursista. O jogo da totalidade e da especialidade torna-se uma prática na formação em tecnologias digitais acessíveis. Cada professor cursista explora um conjunto de recursos e estratégias técnicopedagógicas inclusivas, mas focaliza seu estudo na área da deficiência que, no momento, se coloca como maior desafio de docência.

A flexibilização curricular foi interpretada positivamente por $94 \%$ dos alunos, em geral, e por $100 \%$ dos alunos com deficiência. A análise das respostas discursivas relacionadas ao tema deflagrou a emergência de sete elementos que contribuem para a qualificação da formação, uma vez que, de acordo com os sujeitos da pesquisa:

- adequa a formação às demandas e necessidades específicas da realidade de cada professor: "O fato de podermos optar por uma das atividades me ajuda a aprender mais sobre determinado assunto ou recurso que estiver precisando para trabalhar com algum aluno";

- amplia o conhecimento na perspectiva da livre exploração: "Quando você tem a opção de escolha, você passa a ter conhecimento de cada alternativa antes para depois optar. Assim, sem querer, o cursista tem um pouco de conhecimento de todas as atividades oferecidas no curso";

- valoriza a diversidade humana ao dar vazão a interesses e habilidades particulares: "tendo a possibilidade de escolher a atividade para ser realizada, o aluno tem a autonomia para fazê-la, de acordo com o seu perfil'";

- agiliza o tempo de que o professor dispõe para realizar o curso: "Poder escolher apenas uma atividade [em cada bloco] é muito bom pelo fato de meu tempo ser reduzido e eu não poder fazer todas as atividades obrigatoriamente";

- promove a autonomia e a corresponsabilidade do professor perante a formação: "quando nós escolhemos a atividade, temos ainda mais responsabilidade sobre ela";

- adequa o currículo aos recursos tecnológicos de que o professor dispõe: "nos permite realizar as tarefas, adequando-as de acordo com a estrutura que temos acesso em casa e na instituição de trabalho", e

- valoriza os conhecimentos prévios do professor em determinadas áreas: "possibilita a aluno utilizar seus conhecimentos e ampliar as possibilidades de conhecer novos recursos".

Os posicionamentos negativos à formatação curricular flexível representaram $6 \%$ do total de pessoas que responderam ao questionário, todas sem deficiência alguma. Os elementos mencionados foram: (1) sentimento de desorientação com relação à escolha pela atividade a ser realizada; (2) bate-papos menos eficientes para esclarecimento de dúvidas, uma vez que as orientações são descentralizadas em razão da variedade de atividades oferecidas; (3) sentimento de frustração com relação às atividades que não puderam realizar em virtude da grande oferta e (4) as atividades optativas podem fazer com que alguns professores não ampliem seus conhecimentos, escolhendo entre as opções aquelas com as quais já possuem familiaridade.

Tal percepção pode ser interpretada sob a forte influência da herança de um processo de ensino focado em métodos tradicionais, situação provavelmente vivenciada como realidade formativa entre tais sujeitos, com idade média superior a 41 anos. A 
relutância em abandonar hipóteses metodológicas já consolidadas implica aceitar inovações pedagógicas desafiadoras. Nesse sentido, Montes (2012) coloca que o modelo tradicional traz o conforto do conhecido e do costumeiro às práticas pedagógicas, sendo que, usualmente, o professor abdica de orientar sua docência e, por consequência, seu processo de aprendizagem, por caminhos mais abrangentes, interdisciplinares e complexos.

De acordo com os respondentes, diferentes aspectos contribuem para sua permanência no curso, dentre os quais as questões centrais deste estudo, relacionadas à facilidade de acesso às atividades, à clareza dos conteúdos, flexibilidade, dinâmica e metodologia, elementos esses presentes em 66 fragmentos discursivos. Também foram destacados como aspectos que promovem a permanência dos respondentes no curso questões objetivas como a utilidade do conteúdo do curso em sua prática pedagógica ou mesmo sua validade em relação ao contexto em que se está inserido, em outras palavras, aquilo que seus parâmetros pessoais lhes permitem julgar dialeticamente.

\section{CONSIDERAÇÕES FINAIS}

Os resultados obtidos a partir da análise da percepção de sujeitos com e sem deficiência quanto às estratégias de acessibilidade, técnicas e pedagógicas, propostas pela equipe do NIEE/UFRGS no Curso Tecnologias Digitais Acessíveis evidenciam a importância da implementação de estratégias que abarquem a diversidade humana em contextos formativos, sobretudo daqueles que se operacionalizam a distância. Ao fazer emergir um conjunto de elementos que apoiam o acesso e a permanência de professores cursistas com deficiências, observamos que os conteúdos também se mostram mais adequados ao público que inicialmente não demonstrava variações físicas ou sensoriais.

Nos dois planos em que a acessibilidade do curso foi avaliada (AVA e páginas HTML com descrição das atividades e conteúdo curricular), observou-se significativa redução de barreiras naqueles que foram especialmente produzidos com base nas diretrizes de acessibilidade WCAG 2.0, considerando a diversidade humana e tecnológica. A pesquisa ainda apontou elementos que dão suporte para futuras decisões gerenciais, como estratégias que ampliem a acessibilidade no plano AVA. Em resposta a esse resultado, a equipe do NIEE/UFRGS conduz atualmente um projeto de desenvolvimento de um novo AVA, com base nas diretrizes internacionais de acessibilidade e heurísticas de usabilidade.

No que diz respeito às adaptações curriculares, reafirmou-se a efetividade da estratégia de flexibilização adotada que, ao promover a aproximação do conteúdo à realidade dos professores cursistas, passa a oportunizar a corresponsabilidade de cada sujeito por seu processo formativo. $\mathrm{O}$ aprimoramento de técnicas que explicitem o cuidado com questões relacionadas à acessibilidade em ambientes digitais, muito além de alinhar a filosofia de um curso que aborda a temática inclusiva à sua prática pedagógica, contribui para a concretização de políticas públicas que estabelecem como direito do cidadão o acesso à Educação em todas as suas esferas. 


\section{REFERÊNCIAS}

BARDIN, L. Análise de conteúdo. Lisboa: Ed. 70, 2007.

BASSANI, P.B.S.; BEHAR, P.A.; HEIDRICH, R. de O.; BITTENCOURT, A.; ORTIZ, E. Usabilidade e acessibilidade no desenvolvimento de interfaces para ambientes de educação à distância. Revista Novas Tecnologias na Educação - RENOTE, V. 8 No 1, 2010.

BASTIEN, J. M. C.; SCAPIN, D. L. Ergonomic Criteria for the Evaluation of HumanComputer Interfaces (version 2.1). Technical Report. May 1993. Paris: INRIA Rocquencourt, 1993.

BURGSTAHLER, S. Real Connections: making distance learning accessible to everyone. Washington University, Seatle. Report, 2001.

BURGSTAHLER, S.; CORRIGAN, B.; MCCARTER, J. Making distance learning courses accessible to students and instructors with disabilities: a case study. Internet and Higher Education. v. 7, n. 3, p. 233-246, 2004.

ESTABEL, L.B.; MORO, E.L. da S. Capacitação de bibliotecários com limitação visual pela educação a distância em ambientes virtuais de aprendizagem. Ci. Inf., Brasília, v. 35, n. 3, p. 209-217, set./dez. 2006

GIL, Antônio Carlos. Como elaborar projetos de pesquisa. São Paulo: Atlas, 2002.

MONTES, Marta Teixeira do Amaral. Implicações da aprendizagem colaborativa para a docência online. Dissertação (Mestrado em Educação) - Universidade Estácio de Sá, 2012.

MORAES, R.. Uma tempestade de luz: a compreensão possibilitada pela análise textual discursiva. Ciência Educação, Bauru, SP, v. 9, n. 2, p. 191-210, 2003

NIELSEN, J. Heuristic Evaluation. In: NIELSEN, J.; MACK, R. L. Usability Inspection Methods. New York: John Wiley \& Sons, 1994.

NIELSEN, J. Usability Engineering. Boston: Academic Press, 1993.

PREECE, J.; Rogers, I.; Sharp, H. Design de Interação: Além da Interação HumanoComputador; Porto Alegre: Bookman, 2005

REZENDE, F. A. Características do ambiente virtual construcionista de ensino e aprendizagem na formação de professores universitários. Dissertação de Mestrado Departamento de Multimeios, Unicamp, 2004.

SANTAROSA, L.M.C.; PASSERINO, L.; BASSO, L. de o.; DIAS, C. de O. Acessibilidade em Ambientes de Aprendizagem por Projetos1: construção de espaços 
virtuais para inclusão digital e social de PNEEs. Revista Novas Tecnologias na Educação

- RENOTE, V.5 Nº 1, 2007.

SONZA, A.P.; SANTAROSA, L.M.C. Ambientes Digitais Virtuais: Acessibilidade aos Deficientes Visuais. Revista Novas Tecnologias na Educação - RENOTE, v.1, $\mathrm{n}^{\mathbf{o}}$ 1, 2003.

THATCHER, J. et al. Web Accessibility: Web Standards and Regulatory Compliance. New York: Friendsoft, 2006.

UNESCO. Coordenadoria Nacional para Integração da Pessoa Portadora de Deficiência. Declaração de Salamanca de Princípios, Políticas e Práticas das Necessidades Educativas Especiais e uma Linha de Ação sobre Necessidades Educativas Especiais. Brasília, 1994.

VALENTE, J. A. Visão analítica da Informática na educação no Brasil: a questão da formação do professor. Revista Brasileira de Informática na Educação. RS: Sociedade Brasileira de Computação, n. 1, setembro de 1997.

VYGOTSKY, L. S. A formação social da mente: o desenvolvimento dos processos psicológicos superiores. $5^{\mathrm{a}}$ ed. São Paulo: Martins Fontes, 1994.

W3C - World Wide Web Consortium. Web Content Accessibility Guidelines 2.0. 2008. Disponível em: < http://www.w3.org/TR/WCAG20/ > Acesso em: jan. 2014. Acesso em: $14 / 4 / 2014$. 